\title{
PROPRIEDADES FUNCIONAIS-DIGESTIVAS E NUTRICIONAIS DE POLPA-REFINADA DE MAÇÃ
}

\author{
Dorivaldo da Silva Raupp ${ }^{1 *}$; Kelly Cristina Rebonato Carrijo²; Léa Luzia Freitas Costa ${ }^{3}$; Sandra \\ Denise Camargo Mendes ${ }^{3}$; David Ariovaldo Banzatto ${ }^{4}$ \\ ${ }^{1}$ Depto. de Zootecnia e Tecnologia de Alimentos - UEPG - Bloco F - CEP: 84030-000 - Ponta Grossa, PR. \\ ${ }^{2}$ Graduanda do Depto. de Zootecnia e Tecnologia de Alimentos, UEPG. \\ ${ }^{3}$ Pós-Graduandas do Depto. de Ciência e Tecnologia de Alimentos, UFSC - Av. Ademar Gonzaga, 1346 - \\ CEP: 88034-001- Florianópolis, SC. \\ ${ }^{4}$ Depto. de Ciência do Solo e Engenharia Agrícola, UEPG. \\ *Autor correspondente <raupp@uepg.br>
}

\begin{abstract}
RESUMO: Propriedades funcionais-digestivas e nutricionais da polpa-refinada-desidratada de maçã (PRM) foram avaliadas em ratos Wistar, tendo como padrão de fibra o farelo de trigo (FT). A PRM apresentou 91,91\% de fibra alimentar/dietária, mais que o dobro do FT (43,69\%), sendo 82,27\% de fração insolúvel e 9,64\% da fração solúvel; $8,20 \%$ de proteínas; $0,57 \%$ de lipídeos; $2,04 \%$ de cinzas (minerais); e ausência de carboidratos digestíveis. A incorporação na dieta dos ratos de PRM ou do padrão FT em proporções de 5\%, 15\% ou 25\% produziu, nos animais, efeitos funcionais-digestivos e nutricionais próprios de fibra alimentar insolúvel. Na concentração de $5 \%$ (PRM e FT forneceram 4,6\% e 2,2\% de fibra alimentar, respectivamente) as duas fontes de fibra produziram, com exceção da densidade de fezes seca, efeitos semelhantes. No entanto, em concentrações de $15 \%$ ou $25 \%$, a PRM resultou em mais defecações, maior peso de fezes seca e densidade, porém, produziu fezes seca de menor volume. Com base no resultado da pesquisa, bem como na quantidade industrial disponível, conclui-se que a "polpa-refinada" de maçã poderá constituir-se em fonte alternativa potencial de fibra alimentar para a formulação de alimentos para consumo em dieta normal, mas, principalmente para alimentos especiais que devem apresentar propriedades funcionais-digestivas relacionadas à fibra alimentar. Palavras-chave: maçã, polpa-refinada, fibra alimentar/dietária, farelo de trigo
\end{abstract}

\section{DIGESTIVE-FUNCTIONAL AND NUTRITIONAL PROPERTIES OF REFINED APPLE POMACE}

\begin{abstract}
This study aimed to determine some digestive-functional and nutritional properties from driedrefined apple pomace (AP). Wheat bran (WB), a conventional dietary fiber source currently used in human nutrition, was considered as a standard for comparison. Total dietary fiber, including the insoluble and soluble fractions of AP were $91.91 \%, 82.27 \%$ and $9.64 \%$, respectively. Other nutrients were protein $(8.20 \%)$, lipid $(0.57 \%)$ and minerals $(2.04 \%)$. Dietary fiber contents in standard WB was $43.69 \%$, a value less than a half of AP. Digestive-functional and nutritional properties of each AP or WB were similar only in the proportion of $5 \%$ of fiber source in the feed, except for faeces density. High proportion in the rat feed of $15 \%$ or $25 \%$ resulted in significant differences, AP having the greatest effects on defecation frequency, faecal weight and density, while WB had the greatest effect on the faecal bulk. The quantity available in the apple industry and the quality of the dried-refined apple pomace evaluated from in vitro and in vivo determinations suggest a use as an edible fiber for humans. This new fiber source can be incorporated into both, normal and digestive-functional food products. Key words: apple, refined pomace, dietary fiber, wheat bran
\end{abstract}

\section{INTRODUÇÃO}

O descarte sólido produzido na indústria de maçã é a matéria sólida despectinada ou não, que permanece depois da extração do suco, e apresenta alta proporção de carboidratos insolúveis, quantidades menores de proteína, minerais e algum suco remanescente, contendo açúcares e outras substâncias solúveis (Walter et al., 1985; Chen et al., 1988a; Wang \& Thomas, 1989; Wosiacki et al., 1989, 1995, 1996; Renard \& Thibault, 1991; Saura Calixto, 1993; Carson et al., 1994).

Wang \& Thomas (1989) determinaram os constituintes da matéria sólida desidratada, porção insolúvel da maçã e remanescente da extração do suco, através da ultrafiltração em membrana, e encontraram em peso seco, 33,2-35,3\% de fibra dietária/alimentar; 20,5$29,2 \%$ de fibra detergente neutro; $24,4-25,1 \%$ de fibra detergente ácido; 3,7-6,1\% de pectina; $16,4-16,7 \%$ de celulose; $4,1-4,7 \%$ de hemicelulose e $8,4-8,9 \%$ de lignina. Segundo Walter et al. (1985), Hackler em 1977 fracionou os constituintes da fração fibra detergente neutro da maçã "Red Delicious", e determinou para a celulose $40 \%$, hemicelulose $39 \%$, lignina $9 \%$ e cutina $5 \%$.

A capacidade de hidratação da fibra de maçã foi também avaliada. Chen et al. (1988a) determinaram que a fibra de maçã foi mais higroscópica e apresentou maior hidratação do que o farelo de trigo e de aveia. Os autores atribuíram tais diferenças a vários fatores: (a) estrutura das 
matérias constituintes da parede celular; (b) maior conteúdo de fibra do produto fibra de maçã; e, (c) menor tamanho das partículas do produto fibra de maçã. Com relação ao efeito do processamento, Renard \& Thibault (1991), determinaram que o processo de despectinização aplicado à polpa de maçã produziu uma matéria sólida que apresentou menor grau de hidratação.

No Brasil, cerca de $30 \%$ da produção de maçã são industrializadas para aproveitamento de sidra, suco e geleia (Wosiacki et al., 1993), sendo que nos últimos anos o setor tem sido estimulado devido ao maior consumo destes produtos alimentícios. A matéria sólida produzida nestes processos é normalmente doada aos pecuaristas da região ou descartada, usualmente nos arredores da indústria processadora.

A indústria brasileira de processamento da maçã tem mostrado interesse em alternativas econômicas e tecnologicamente viáveis para a utilização do descarte sólido produzido. Apesar disso, e enquanto a comunidade científica não responder satisfatoriamente aos anseios destas indústrias, estes produtos continuam a ser considerados, embora quantitativamente relevantes, apenas como resíduos, produtos sem valor econômico. A perda de dinheiro, de nutrientes potenciais e de qualidade do ar nas regiões de processamento é marcante, uma vez que o resíduo fermenta e exala odores pouco agradáveis. Há necessidade, pois, de que se procure caracterizar as matérias sólidas produzidas durante o processamento de suco e de sidra, e se busque alternativas de uso com finalidades mais nobres do que aquelas que vêm sendo praticadas nas regiões de industrialização de maçã (Raupp et al., 1999).

Cumpre observar, todavia, que pesquisadores brasileiros, como Wosiacki et al. (1989, 1993, 1995, 1996), Namiuchi et al. (1992) vêm, já há algum tempo, desenvolvendo estudos que compreendem a melhoria de qualidade para o setor de cultivo e de industrialização da maçã; entretanto, pouco tem sido feito em relação ao aproveitamento das matérias sólidas, como a polpa refinada, bem como de outros resíduos da industrialização desta fruta, para fins de uso na alimentação humana.

Algumas propostas visando aproveitar, para fins alimentícios, a matéria sólida produzida nas unidades industriais de maçã, foram desenvolvidas por pesquisadores da comunidade científica mundial, incluindo a do Brasil, e, na maioria, foram determinadas a caracterização química e física, mas, também avaliou-se o aproveitamento dessa matéria sólida como ingrediente de alimentos formulados, como para os produtos da panificação e outros; em contraste, muito pouco tem sido feito com relação a avaliação de suas propriedades in vivo, funcionais-digestivas e nutricionais (Walter et al., 1985; Hang, 1987; Chen et al., 1988a,b; Wang \& Thomas, 1989; Renard \& Thibault, 1991; Saura Calixto, 1993; Carson et al., 1994; Protzek et al., 1998).

A fibra alimentar (fibra dietária), definida recentemente como o somatório de polissacarídeos e substâncias relacionadas indigeríveis mais a lignina, é o constituinte de alimentos e de produtos alimentícios que resiste à hidrólise dos sucos digestivos do homem. A fibra ingerida na alimentação humana não é hidrolisada até o intestino delgado, mas, já a partir da porção terminal do intestino delgado e, principalmente, ao alcançar o intestino grosso, a fração solúvel é extensamente fermentada pela flora natural microbiana, enquanto que, a fração insolúvel permanece quase que totalmente intacta. Em humanos, cerca de $70 \%$ da fibra ingerida na dieta é fermentada. A evolução do conhecimento científico permitiu concluir que a normalidade digestiva, bem como, a prevenção e o tratamento de doenças como a constipação, a diverticulite, a hipercolesterolemia, a hiperglicemia, a obesidade, o câncer do intestino grosso e da mama, estão relacionados, em parte, à ingestão de fibra alimentar. Contudo, os diversos constituintes da fibra alimentar não têm o mesmo efeito ou ação fisiológica. A fibra solúvel contribui para o aumento do volume intra-luminal, no entanto, tem pouco efeito, em contraste com a fibra insolúvel, no peso e no volume fecal (Mikkelsen et al., 1979; Bolton et al., 1981; Smith et al., 1981; Anderson \& Bryant, 1986; Schneeman, 1987; Roehrig, 1988; Seva-Pereira et al., 1991; Schweizer \& Edwards, 1992; Hernandez et al., 1995; Raupp \& Sgarbieri, 1997).

As fibras possuem, também, a capacidade de complexar-se com outros constituintes da dieta através de vários mecanismos, podendo arrastá-los em maior quantidade na excreção fecal. Dessa forma, as substâncias tóxicas, mas também nutrientes, podem ser excretados em maior ou menor quantidade, dependendo da fibra presente na dieta (Walker, 1975; Cummings, 1978; Toma \& Curtis, 1986; Davies et al., 1991; Oliveira et al., 1991; Raupp \& Sgarbieri, 1996).

Pelo exposto, ficou evidente que, embora subutilizados, os descartes produzidos pelas indústrias de maçã já estão sendo aproveitados, com vantagens, para a alimentação de animais e, embora em baixa proporção, algumas propostas já sugerem seu uso para a alimentação humana.

É nesse contexto que outros estudos com essa matéria alimentícia potencial estão sendo desenvolvidos no Laboratório de Tecnologia de Alimentos da Universidade Estadual de Ponta Grossa, em particular, com relação as suas propriedades funcionais-digestivas e nutricionais, e tendo por fim o seu uso também para a alimentação humana.

A pesquisa em foco estudou a matéria sólida refinada-desidratada resultante da extração de suco da maçã, a qual é considerada quase na sua totalidade como descarte na indústria de suco e de sidra, e buscou três objetivos básicos:

a) Caracterização, em termos de composição centesimal, granulometria e capacidade de hidratação da polpa de maçã refinada-desidratada;

b) Avaliação de algumas das propriedades funcionais-digestivas e nutricionais, em ratos modelo Wistar, da polpa de maçã refinada-desidratada; 
c) Em sendo promissores os resultados da pesquisa, propor o uso desse descarte, também, como fonte de nutriente e como ingrediente de alimentos, principalmente para aqueles alimentos fibrosos formulados e destinados à alimentação humana. Assim, a pesquisa estará contribuindo, por conseguinte, para solucionar a despoluição do meio-ambiente, já que, esse descarte das indústrias de maçã, se jogados no solo, passam a ser, com o tempo, agentes de poluição do ar e das águas.

\section{MATERIAL E MÉTODOS}

Amostra de polpa de maçã despectinada e úmida foi produzida no Laboratório de Tecnologia de Alimentos da Universidade Estadual de Ponta Grossa e, de modo geral, assemelhada ao procedimento utilizado na indústria de suco de maçã. A maçã foi cortada em fatias finas e esmagada em prensa de controle manual para extração do suco. A seguir, a matéria sólida úmida foi triturada utilizando-se o processador industrial de alimentos juntamente com um preparado enzimático contendo enzimas pectinolíticas. Depois de cerca de 3 horas, o material foi filtrado e a matéria retida no filtro foi submetida à lavagem com água corrente sobre peneira, começando com $2 \mathrm{~mm}$ e até $0,1 \mathrm{~mm}$, para esgotamento de açúcares solúveis. A matéria sólida foi colocada, em espessura reduzida ao máximo, sobre lonas de plástico escuro, onde ficou exposta à radiação solar durante aproximadamente 30 horas. $\mathrm{A}$ polpa refinada e desidratada foi triturada em processador industrial de alimentos em sua máxima rotação por períodos intermitentes e até somar 10 minutos; a seguir, foi peneirada para obtenção de um produto farináceo denominado de polpa-refinada de maçã (PRM), a qual foi mantida em embalagem hermética até a sua utilização nas determinações analíticas e nos ensaios biológicos.

Na polpa-refinada de maçã (PRM) e no padrão farelo de trigo (FT) adquirido no comércio, foi determinada a composição centesimal utilizando-se os métodos descritos na AOAC (1984) para a umidade, proteína bruta, matéria graxa, cinza (totalidade de minerais que permanecem depois da incineração da amostra alimentícia), e a fibra alimentar/dietária pelo método de Prosky et al. (1988). Os carboidratos digeríveis foram estimados no hidrolisado produzido no procedimento analítico de determinação da fibra alimentar e utilizando o método de Somogyi (Somogyi, 1945).

A capacidade de rehidratação foi determinada segundo o procedimento relatado por Schweizer \& Edwards (1992). A granulometria foi determinada através de peneiramento de $100 \mathrm{~g}$ de amostra durante 30 minutos, e usando-se peneiras de 2 - 1 - 0,5 - 0,25-0,105 mm. As partículas constituintes da amostra e retidas nas peneiras foram pesadas e calculadas as suas proporções no produto.

A PRM foi usada no preparo de dietas destinadas à alimentação de ratos Wistar em crescimento para avaliação de suas propriedades funcionais-digestivas e nutricionais, e tendo o FT como padrão de fibra. O ensaio consistiu de sete tratamentos de seis ratos/tratamento e os ratos foram distribuídos entre os tratamentos segundo o modelo de blocos ao acaso. Três tratamentos receberam a PRM nas concentrações de $5 \%, 15 \%$ ou $25 \%$; três tratamentos receberam FT como padrão de comparação também nas proporções de $5 \%, 15 \%$ ou $25 \%$; e, um tratamento não recebeu fonte de fibra. Para o preparo das dietas utilizou-se ingredientes de diversas procedências: caseína 60 mesh fornecida pela Kauffmann \& Co., contendo $90,39 \%(\% \mathrm{~N} \times 6,40)$ de proteína, em base seca; óleo refinado de soja; amido de milho fornecido pelo produto comercial "maizena" de grau de pureza igual a $99,8 \%$, em base seca; mistura de vitaminas e mistura de sais minerais, preparadas em laboratório segundo a formulação da AIN-93G (Reeves et al., 1993). A dieta básica foi preparada segundo a formulação da AIN-93G (Reeves et al., 1993) a qual contem $20 \%$ de caseína; $7 \%$ de óleo de soja; $3,5 \%$ de mistura mineral; $1 \%$ de mistura vitamínica; $0,3 \%$ de L-cistina; $0,25 \%$ de bitartarato de colina. $\mathrm{O}$ amido foi a única fonte de carboidrato digerível e entrou na dieta básica em q.s.p. 100\%. A PRM ou 0 padrão FT entraram, como única fonte de fibra alimentar, na formulação das dietas para os ratos nas proporções de $5 \%, 15 \%$ ou $25 \%$, e em substituição a igual porcentagem de amido. A formulação da AIN-93G (Reeves et al., 1993) fornece $5 \%$ de fibra para a dieta básica.

Os animais modelo utilizados foram ratos albinos da linhagem Wistar, SPF (Livres de Patógenos Específicos), de 21 dias e fornecidos pelo Biotério Central da Universidade Federal de Santa Catarina (UFSC). Durante o ensaio, realizado no Laboratório de Ensaios Biológicos da Pós-Graduação em Ciência de Alimentos, UFSC, a temperatura do laboratório foi mantida a $21 \pm 2^{\circ} \mathrm{C}$, com alternância automática de claro-escuro em períodos de 12 horas. Os animais receberam dieta e água ad libitum. $O$ ensaio teve duração de 33 dias, incluindo 5 dias de adaptação ao ambiente de experimentação e 28 dias para coleta de dados, durante os quais foram feitas, para cada rato, as determinações de: peso corpóreo; peso de alimento ingerido; volume de água ingerida; peso de fezes seca; volume de fezes seca; número de defecações; bem como, foram calculados: densidade de fezes seca; quociente de eficiência alimentar (QEA); quociente de eficiência protéica operacional (PERop).

Os dados foram analisados estatisticamente utilizando-se o programa ESTAT desenvolvido no Polo Computacional da Faculdade de Ciências Agrárias e Veterinárias, UNESP, Jaboticabal. Foi realizada a análise de variância com desdobramento por contrastes que fez comparações específicas entre grupos de tratamentos e seguiu o modelo descrito a seguir: $Y(1)$ - Tratamento sem fonte de fibra vs. Tratamentos contendo fonte de fibra; $Y$ (2) - Tratamento contendo $5 \%$ de PRM vs. Tratamento contendo $5 \%$ de FT; Y (3) - Tratamento contendo $15 \%$ de PRM vs. Tratamento contendo $15 \%$ de FT; Y (4) Tratamento contendo 25\% de PRM vs. Tratamento contendo $25 \%$ de FT. Também, os dados foram avaliados 
à luz da análise de regressão polinomial para ajuste de uma função estatística que relacione as características (variáveis) em função dos níveis de fonte de fibra alimentar utilizados nas dietas.

\section{RESULTADOS E DISCUSSÃO}

O produto "polpa-refinada e desidratada de maçã" foi avaliado tendo por fim o seu uso como ingrediente e fonte de fibra alimentar, em particular, para alimentos especiais formulados, os chamados de "alimentos funcionais fibrosos", mas, também como ingrediente para alimentos de consumo em dieta normal.

Os resultados da composição centesimal, hidratação e granulometria da polpa-refinada de maçã (PRM) bem como do padrão, farelo de trigo (FT), estão apresentados nas TABELAS 1, 2 e 3, respectivamente.

O conteúdo super-alto (TABELA 1) igual a 91,91\% de fibra alimentar/dietária, sendo $82,27 \%$ de fração insolúvel e apenas 9,64\% da fração solúvel, para a PRM, torna esse produto atraente para uso na alimentação humana. No padrão de comparação, farelo de trigo (FT), uma fonte convencional de fibra comestível, foi determinado $43,69 \%$ para o constituinte fibra alimentar, menos da metade do valor que possui a PRM.

Baixas quantidades foram determinadas para os outros nutrientes constituintes da PRM (TABELA 1): 8,20\% de proteína (\%N x 6,25); 0,57\% de lipídeo; 2,04\% de cinza; e, ausência de carboidrato-digerível. Em contraste, no FT foram determinadas quantidades expressivas iguais a $19,79 \%-3,43 \%-6,33 \%$, respectivamente, para proteína - lipídeo - cinza, e 27,92\% para carboidrato-digerível.

Valores bastante diferenciados foram determinados por Chen et al. (1988a), Wang \& Thomas (1989), Renard \& Thibault (1991), Saura Calixto (1993), Carson et al. (1994) para a fibra dietária/alimentar, a fração insolúvel e a fração solúvel, os quais variaram de 33,2$88,6 \%, 29,9-78,3 \%$ e 3,0-22,5\%, respectivamente, e foram influenciados pela variedade da maçã, pelo processamento e o método analítico aplicado.

A maior proporção (TABELA 1) de fibra alimentar da PRM pode explicar o seu maior grau de hidratação (TABELA 2) comparado com o padrão FT. O volume da PRM aumentou em 3 e 3,8 vezes quando foi deixado submerso em água sem e com agitação, respectivamente, enquanto que, o volume do FT aumentou menos, respectivamente, 2 e 2,3 vezes.
Segundo Chen et al. (1988a) o produto preparado de fibra de maçã apresentou maior capacidade de hidratação do que o farelo de trigo, propriedade atribuída à estrutura das matérias constituintes da parede celular, ao maior conteúdo de fibra e ao menor tamanho das partículas do produto fibra de maçã.

Quase a totalidade, proporção igual a 92,09\%, das partículas da fonte de fibra PRM apresentou tamanho entre 1,0 e $0,105 \mathrm{~mm}$, enquanto que, no padrão FT proporções assemelhadas, $91,48 \%$, de partículas tiveram tamanho um pouco menor, entre 0,5 e 0,105 mm (TABELA 3).

Os efeitos da PRM na freqüência das defecações e nas características das fezes bem como nos parâmetros nutricionais, considerados de importância em qualquer estudo de um produto fibroso, foram determinados através de ensaios biológicos com ratos modelo e tendo como padrão de comparação o FT obtido do comércio. Os resultados estão apresentados nas TABELAS 4 e 5.

TABELA 2 - Grau de hidratação, com agitação e sem agitação, das fontes de fibra alimentar utilizadas na formulação de dietas para os ratos.

\begin{tabular}{lccccc}
\hline \multirow{2}{*}{ Procedimento } & \multicolumn{2}{c}{$\begin{array}{c}\text { Polpa-refinada de } \\
\text { maçã }\end{array}$} & & \multicolumn{2}{c}{ Farelo de trigo ${ }^{3}$} \\
\cline { 2 - 3 } & Início & Final & & Início & Final \\
\hline Sem agitação & 30 & 90 & & 30 & 60 \\
Com agitação & 30 & 114 & & 30 & 68 \\
\hline
\end{tabular}

${ }^{1}$ média de três determinações analíticas; ${ }^{2}$ matéria sólida que sobra depois da extração do suco, seguida da lavagem com água, desidratada pela exposição direta à luz solar e triturada em processador de alimentos; ${ }^{3}$ adquirido no comércio.

TABELA 3 - Granulometria $(p / p)^{1}$ das fontes de fibra alimentar utilizadas na formulação de dietas para os ratos.

\begin{tabular}{lcc}
\hline $\begin{array}{l}\text { Tamanho de } \\
\text { partículas }\end{array}$ & $\begin{array}{c}\text { Polpa-refinada } \\
\text { de maçã }\end{array}$ & Farelo de trigo ${ }^{3}$ \\
\hline $\mathrm{mm}$ & 0,00 & 0,00 \\
$>2,0$ & 1,65 & 0,38 \\
2,0 a 1,0 & 42,35 & 6,15 \\
1,0 a 0,5 & 30,40 & 55,65 \\
0,5 a 0,25 & 19,34 & 35,83 \\
0,25 a 0,105 & 6,26 & 1,99 \\
\hline 0,105 & & ------- \\
\hline
\end{tabular}

${ }^{1}$ média de três determinações analíticas, utilizando amostra de 100 $\mathrm{g} ;{ }^{2}$ matéria sólida que sobra depois de extraído o suco na presença de enzimas pectinolíticas, seguida de lavagem com água, secagem ao sol e triturada em processador de alimentos; ${ }^{3}$ adquirido no comércio.

TABELA 1 - Composição centesimal (base seca) ${ }^{1}$ das fontes de fibra alimentar utilizadas na formulação de dietas para os ratos.

\begin{tabular}{|c|c|c|c|c|c|}
\hline Fonte de fibra & Fibra alimentar & Carbo idrato digerível ${ }^{5}$ & Prote ína & Lipídio & Cinza \\
\hline Polpa-refinada de maçã ${ }^{2}$ & $91,91^{4}$ & 0,00 & 8,20 & 0,57 & 2,04 \\
\hline Farelo de trig $0^{3}$ & 43,69 & 27,92 & 19,79 & 3,43 & 6,33 \\
\hline
\end{tabular}

${ }^{1}$ média de três determinações analíticas; ${ }^{2}$ matéria sólida que sobra depois de extraído o suco na presença de enzimas pectinolíticas, seguida de lavagem com água, secagem ao sol e triturada em processador de alimentos; ${ }^{3}$ adquirido no comércio; ${ }^{4}$ proporção de $82,27 \%$ de fibra insolúvel e 9,64\% de fibra solúvel; ${ }^{5}$ estimado no hidrolisado produzido no procedimento analítico de determinação da fibra alimentar. 
TABELA 4 - Análise de regressão relacionando variáveis dependentes com as concentrações (5, 15 ou 25\%) de fonte de fibra na dieta para os ratos.

\begin{tabular}{|c|c|c|c|}
\hline Variável dependente & Fonte de fibra & Equação de regressão ${ }^{1}$ & $\left(r^{2}\right)$ \\
\hline$N^{\circ}$ de defecações & $\begin{array}{l}\text { PRM } \\
\text { FT }\end{array}$ & $\begin{array}{l}Y=440,44+62,52 X \\
Y=452,60+49,53 X\end{array}$ & $\begin{array}{l}0,9870 \\
0,9774\end{array}$ \\
\hline Peso (g) de fezes seca & $\begin{array}{l}\text { PRM } \\
\text { FT }\end{array}$ & $\begin{array}{l}Y=11,04+2,203 X \\
Y=1018+1744 X\end{array}$ & $\begin{array}{l}0,9936 \\
0,9997\end{array}$ \\
\hline Volume $(\mathrm{mL})$ de fezes seca & $\begin{array}{l}\text { PRM } \\
\text { FT }\end{array}$ & $\begin{array}{l}Y=8,94+5,212 X \\
Y=7,18+6,788 X\end{array}$ & $\begin{array}{l}0,9973 \\
0,9970\end{array}$ \\
\hline Densidade das fezes seca & $\begin{array}{l}\text { PRM } \\
\text { FT }\end{array}$ & $\begin{array}{l}Y=0,8266+0,01592 X \\
Y=0,7528+0,0209 X\end{array}$ & $\begin{array}{l}0,9336 \\
0,8123\end{array}$ \\
\hline Ganho de peso (g) corpóreo & $\begin{array}{l}\text { PRM } \\
\text { FT }\end{array}$ & $\begin{array}{l}\text { Não houve ajuste } \\
\text { Não houve ajuste }\end{array}$ & $\begin{array}{l}- \\
-\end{array}$ \\
\hline Alimento ingerido $(\mathrm{g})$ & $\begin{array}{l}\text { PRM } \\
\text { FT }\end{array}$ & $\begin{array}{l}\text { Não houve ajuste } \\
\text { Não houve ajuste }\end{array}$ & - \\
\hline Água ingerida $(\mathrm{mL}) / 100 \mathrm{~g}$ de alimento ingerido & $\begin{array}{l}\text { PRM } \\
\text { FT }\end{array}$ & $\begin{array}{l}\text { Não houve ajuste } \\
\text { Não houve ajuste }\end{array}$ & - \\
\hline Quociente de eficiência alimentar (QEA) & $\begin{array}{l}\text { PRM } \\
\text { FT }\end{array}$ & $\begin{array}{l}\text { Não houve ajuste } \\
\text { Não houve ajuste }\end{array}$ & $\begin{array}{l}- \\
-\end{array}$ \\
\hline $\begin{array}{l}\text { Quociente de eficiência protéica peracional } \\
\text { (PERop) }\end{array}$ & $\begin{array}{l}\text { PRM } \\
\text { FT }\end{array}$ & $\begin{array}{l}\text { Não houve ajuste } \\
\text { Não houve ajuste }\end{array}$ & - \\
\hline
\end{tabular}

PRM=matéria sólida resultante de extração do suco na presença de enzimas pectinolíticas, seguida de filtração, lavagem com água, secagem ao sol e triturado em processador de alimentos; FT=padrão farelo de trigo; ${ }^{1} 0 \leq X \leq 25 \%$; $\left(r^{2}\right)=$ coeficiente de determinação.

TABELA 5 - Média do(s) Tratamento(s) e resultado da análise de variância com desdobramento por contrastes (significância de F) para as variáveis medidas no ensaio biológico com ratos.

\begin{tabular}{|c|c|c|c|c|c|c|c|c|c|c|c|c|}
\hline \multirow{3}{*}{ Variável } & \multicolumn{12}{|c|}{ Tratamento } \\
\hline & \multirow{2}{*}{$\begin{array}{l}\text { Sem } \\
\text { f.fibra }\end{array}$} & \multirow{2}{*}{$\begin{array}{c}\text { Com } \\
\text { f.fibra }\end{array}$} & \multirow[t]{2}{*}{$\mathrm{F}$} & \multicolumn{3}{|c|}{$5 \%$} & \multicolumn{3}{|c|}{$15 \%$} & \multicolumn{3}{|c|}{$25 \%$} \\
\hline & & & & PRM & FT & $\mathrm{F}$ & PRM & FT & $\mathrm{F}$ & PRM & FT & $\mathrm{F}$ \\
\hline No de defecações & 375 & 1311 & ** & 786 & 752 & n.s. & 1475 & 1285 & n.s. & 1939 & 1627 & * \\
\hline Peso (g) de fezes seca & 10,1 & 40,6 & ** & 21,8 & 19,5 & n.s. & 46,9 & 36,9 & $\star *$ & 64,5 & 53,8 & ** \\
\hline Volume $(\mathrm{mL})$ de fezes seca & 11,7 & 96,9 & ** & 30,8 & 35,8 & n.s. & 88,7 & 108,3 & * & 139,2 & 178,3 & ** \\
\hline Densidade das fezes seca & 0,87 & 0,49 & ** & 0,72 & 0,55 & ** & 0,54 & 0,35 & ** & 0,47 & 0,30 & ** \\
\hline Ganho de peso (g) corpóreo & 103,5 & 140,8 & ** & 152,4 & 134,0 & n.s. & 144,6 & 134,9 & n.s. & 146,7 & 132,3 & n.s \\
\hline Alimento ing erido $(\mathrm{g})$ & 320,6 & 69,8 & * & 378,9 & 328,5 & n.s. & 378,3 & 352,2 & n.s. & 406,6 & 374,2 & n.s \\
\hline $\begin{array}{l}\text { Água ingerida }(\mathrm{mL}) / 100 \mathrm{~g} \text { de } \\
\text { alimento ingerido }\end{array}$ & 199,7 & 161,0 & * & 138,6 & 147,9 & n.s. & 182,7 & 168,0 & n.s. & 153,2 & 176,1 & n.s \\
\hline $\begin{array}{l}\text { Quociente de eficiência } \\
\text { alimentar (QEA) }\end{array}$ & 0,32 & 0,38 & * & 0,40 & 0,41 & n.s. & 0,38 & 0,38 & n.s. & 0,37 & 0,35 & n.s \\
\hline $\begin{array}{l}\text { Quociente de eficiência protéica } \\
\text { operacional (PERop) }\end{array}$ & 1,87 & 2,24 & * & 2,32 & 2,41 & n.s. & 2,22 & 2,21 & n.s. & 2,13 & 2,04 & n.s \\
\hline
\end{tabular}

PRM=matéria sólida resultante de extração do suco na presença de enzimas pectinolíticas, seguida de filtração, lavagem com água, secagem ao sol e triturado em processador de alimentos; $F T=$ padrão farelo de trigo; *significativo para $p<0,05$; ${ }^{* *}$ significativo para $p<$ 0,$01 ;$ n.s. $=$ não significativo ( $p>0,05$ ); modelo: $Y 1$, T. sem fonte de fibra vs T. contendo $5 \%, 15 \%$ ou $25 \%$ de fonte de fibra - Y2, T. contendo $5 \%$ de PRM vs T. contendo $5 \%$ de FT - Y3, T. contendo $15 \%$ de PRM vs T. contendo $15 \%$ de FT - Y4, T. contendo $25 \%$ de PRM vs T. contendo $25 \%$ de FT.

A TABELA 4 mostra o resultado da análise de regressão polinomial, aplicada aos dados obtidos do ensaio biológico, para ajuste de uma função estatística (regressão linear, regressão quadrática, regressão cúbica) que relacione as diversas variáveis estudadas em função da incorporação na dieta de $5 \%, 15 \%$ ou $25 \%$ da fonte de fibra PRM ou do padrão FT. Esta análise mostrou existir, como esperado, um elevado grau de ajuste segundo o modelo de regressão linear para algumas das variáveis dependentes (TABELA 4), as quais estão relacionadas com as características das fezes e com as defecações. Destas, apenas a densidade das fezes seca do Tratamento com o padrão FT produziu um coeficiente de determinação mais baixo, igual a $81,2 \%$; para os demais casos, o coeficiente esteve acima de 93,0\%.

Assim, conclui-se que, a incorporação na dieta dos ratos modelos de qualquer das duas fontes de fibra, PRM ou $\mathrm{FT}$, em proporções crescentes de $5 \%, 15 \%$ ou $25 \%$, produziram efeitos funcionais-digestivos próprios de fibra alimentar constituída de alta concentração da fração 
insolúvel, os quais seguiram o modelo da regressão linear (TABELA 4) e foram medidos através das variáveis peso, volume e densidade das fezes seca, bem como, a freqüência das defecações dos animais.

Para qualquer das outras variáveis nutricionais dependentes, como alimento ingerido durante o ensaio, água ingerida por $100 \mathrm{~g}$ de alimento ingerido, QEA, PERop e ganho de peso corpóreo (TABELA 4), não foi encontrado um bom grau de ajuste, tanto para a PRM como para o padrão FT. Os dados ou não se ajustaram a nenhum dos três modelos estudados ou o ajuste foi muito baixo, e, portanto, conclui-se que a ingestão de mais fibra não estimulou os ratos a ingerirem mais alimento ou mais água, nem resultou em aumento de peso corpóreo, no QEA e no PERop.

A análise de variância com desdobramento por contrastes (TABELA 5) mostrou, para as variáveis que mediram as características das fezes secas e a freqüência das defecações dos ratos modelos, existir diferença significativa entre o Tratamento que não recebeu a adição de fibra (Tratamento sem fonte de fibra) com os Tratamentos que receberam as fontes de fibra PRM ou FT. A densidade das fezes seca foi menor nos Tratamentos com as fontes de fibra, enquanto que, as demais variáveis apresentaram valores, como esperado, maiores para os Tratamentos que receberam as fontes de fibra.

Também, para as outras variáveis nutricionais, como ganho de peso corpóreo, alimento ingerido, QEA e PERop (TABELA 5), os Tratamentos que receberam as fontes de fibra, PRM ou FT, apresentaram valor significativamente maior que o Tratamento que não recebeu a fonte de fibra, porém, para a variável água ingerida por $100 \mathrm{~g}$ de alimento ingerido o valor foi significativamente menor para os Tratamentos que receberam as fontes de fibra PRM ou FT.

Na TABELA 5, também são mostrados os dados resultantes da avaliação comparativa entre as duas fontes de fibra testadas, a PRM e o padrão FT. A análise de variância com desdobramento por contrastes dos tratamentos mostrou que para as variáveis nutricionais, alimento ingerido, água ingerida por $100 \mathrm{~g}$ de alimento ingerido, ganho de peso corpóreo dos ratos, QEA e $P E R o p$, não houve diferença significativa entre 0 Tratamento que recebeu dieta contendo PRM e o Tratamento que recebeu dieta contendo o padrão FT, para qualquer dos três níveis testados de 5\% (PRM forneceu 4,6\%, FT forneceu 2,2\% de fibra alimentar), 15\% (PRM forneceu $13,8 \%$, FT forneceu $6,6 \%$ de fibra alimentar) ou $25 \%$ (PRM forneceu $23,0 \%$, FT forneceu $11,0 \%$ de fibra alimentar) da fonte de fibra na dieta.

Já, com relação às características de funcionalidade-digestiva do animal modelo, as duas fontes distintas de fibra alimentar produziram alguma diferença significativa nas variáveis estudadas para qualquer concentração da fonte de fibra acrescentada na dieta (TABELA 5). Assim, para as proporções da fonte de fibra acrescentadas na dieta, iguais a $5 \%, 15 \%$ ou $25 \%$, a PRM produziu fezes seca de densidade significativamente maior que o padrão $\mathrm{FT}$.

No entanto, para as variáveis que mediram outras características das fezes, como o peso e o volume, bem como, o número de defecações, não ocorreu diferença significativa entre as duas fontes distintas de fibra (TABELA 5) apenas nos Tratamentos que receberam a menor concentração, igual a $5 \%$, da fonte de fibra na dieta. Para qualquer dos Tratamentos que receberam as concentrações maiores da fonte de fibra na dieta, de $15 \%$ ou de $25 \%$, as quais forneceram fibra alimentar para os ratos em quantidades consideradas por Reeves et al. (1993) acima do normal de 5\%, observou-se que, a PRM produziu número de defecações e fezes de peso significativamente maior, porém, o padrão FT produziu as fezes de maior volume.

Embora não tenha ocorrido diferença significativa entre as duas fontes de fibra para a variável alimento ingerido (TABELA 5), os ratos que receberam a dieta contendo a PRM ingeriram maiores quantidades de fibra alimentar, pois, na fonte PRM foi determinado (TABELA1) mais que o dobro do valor encontrado para a fibra alimentar no FT. Estes dados podem explicar porque a fonte PRM foi a mais eficiente para formar a maior massa fecal nos ratos que receberam dietas contendo proporções altas de $15 \%$ ou de $25 \%$ da fonte de fibra na dieta, bem como, a mais eficiente em estimular o maior número de defecações nos ratos alimentados com dietas contendo a mais alta concentração de $25 \%$ da fonte de fibra.

Em contraste, os ratos que receberam a fonte FT ingeriram menor quantidade de fibra alimentar, ainda assim, produziram fezes de maior volume do que os ratos que receberam a dieta contendo a fonte PRM. Assim, deduz-se que a fibra alimentar do FT mostrou ser mais eficiente do que a da PRM para aumentar o volume das fezes produzidas pelos ratos.

Que a ingestão de fibra insolúvel produz, tanto em animais modelos como em humanos, aumento de peso, de volume, de freqüência das defecações, mas, diminuição da densidade das fezes já foi fartamente documentado na literatura científica específica, e, estão relacionadas com a regulação das funções digestivas bem como com a prevenção e o tratamento de doenças do trato gastrintestinal, como constipação, diverticulite e câncer do cólon.

Saura Calixto (1993) avaliou em ratos modelos o efeito de dieta contendo $10 \%$ de um produto fibroso preparado a partir de maçã, tendo como referência uma outra dieta sem a adição de fibra, e observou que o grupo que recebeu a fibra produziu fezes, como na pesquisa em pauta, também de maior peso, aumento de $240-288 \%$. Mas, em contraste com os resultados aqui produzidos, não ocorreu diferença significativa entre o grupo que recebeu a fibra e o grupo sem a adição de fibra, tanto para o consumo de alimento como para o ganho de peso corpóreo dos ratos. Em estudos com bagaço de mandioca constituído de alta proporção de fibra alimentar insolúvel, Raupp et al. (1998) também demonstraram que ratos alimentados 
com dieta contendo a fonte de fibra insolúvel produziram fezes de maior peso, maior volume, menor densidade, bem como, maior número de defecações, do que os ratos que receberam a dieta isenta da adição de fibra.

No entanto, Raupp \& Sgarbieri (1997) estudaram o efeito nutricional e fisiológico-digestivo de uma fibra solúvel de alta viscosidade também em ratos modelos, e demostraram que esta fibra produziu, em contraste, fezes de peso e volume muito menores, bem como foi menor a freqüência dos animais para defecar, comparativamente à fibra insolúvel celulose purificada.

Estudos que avaliaram o efeito da fibra alimentar em estimular ou inibir o consumo de alimentos e no peso corpóreo também foram relatados. Segundo Raupp \& Sgarbieri (1996), o acréscimo de fibra insolúvel celulose à dieta de ratos em concentração de 3\%,6\% e $9 \%$ estimulou uma maior ingestão de alimentos, mas não afetou significativamente o peso dos ratos. Porém, a fibra solúvel de alta viscosidade (Raupp \& Sgarbieri, 1997) extraída do feijão carioca $80 \mathrm{SH}$ atuou no sentido de diminuir a ingestão da dieta e afetou negativamente 0 ganho de peso corpóreo dos ratos. Redução de peso corpóreo em humanos e em ratos foi observada, também, por Mikkelsen et al. (1979), por Krotkiewski (1984) em indivíduos alimentados, respectivamente, com celulose e goma-guar, e, também, por Davies et al. (1991) o qual forneceu aos ratos dietas contendo fibras purificadas, pectina ou celulose.

Pelo exposto, ficou claro que a fonte teste polparefinada de maçã (PRM) produziu efeitos nas características das fezes e nas defecações de ratos modelos considerados próprios de uma fonte de fibra alimentar insolúvel. No geral, suas propriedades funcionais-digestivas avaliadas nos ratos modelos não foram distintas daquelas produzidas pelo padrão farelo de trigo (FT), uma fonte convencional de fibra alimentar para a alimentação humana. Ainda, seus efeitos nos parâmetros nutricionais, como no ganho de peso corpóreo, na ingestão de alimento, no QEA e no PERop foram semelhantes aos produzidos pelo padrão farelo de trigo.

\section{CONCLUSÕES}

A polpa-refinada de maçã apresentou alta concentração, cerca de $92,0 \%$ na base seca, de fibra alimentar/dietária, mais que o dobro do padrão farelo de trigo, e teve como principal constituinte quantitativo $(82,27 \%$, b.s.) a fração insolúvel da fibra.

A polpa-refinada de maçã incorporada em dieta para alimentar ratos modelos em proporção igual a 5\%, bem como em proporções mais altas de $15 \%$ ou $25 \%$, produziu nos animais efeitos funcionais-digestivos próprios de fibra alimentar insolúvel, os quais foram, no geral, semelhantes aos do padrão farelo de trigo. O quociente de eficiência alimentar, o quociente de eficiência protêica operacional e o ganho de peso corpóreo dos animais foram semelhantes para ambas as fontes de fibra.
A "polpa-refinada de maçã" poderá constituir fonte alternativa potencial do nutriente fibra alimentar para a formulação de alimentos destinados ao consumo humano, principalmente os especiais, que apresentem propriedades específicas relacionadas à fibra alimentar/dietária.

Esta proposta estará contribuindo para a despoluição nas regiões de processamento da sidra e do suco de maçã.

\section{AGRADECIMENTOS}

O primeiro autor agradece ao CNPq/PIBIC/UEPG pela concessão de uma bolsa de iniciação científica para o período ago./96 a jul./97; À Coordenação da PósGraduação em Ciência de Alimentos, UFSC por permitir o uso do Laboratório de Ensaios Biológicos; ao Dr. Gilvan Wosiacki pelo fornecimento das maçãs; e ao Dr. André Belmont Pereira, UEPG por revisar o abstract.

\section{REFERÊNCIAS BIBLIOGRÁFICAS}

ANDERSON, J.W.; BRYANT, C.A. Dietary fiber: diabets and obesity. American Journal of Gastroenterology, v.81, p.898-906, 1986. ASSOCIATION OF OFFICIAL ANALYTICAL CHEMISTS. Official methods of analysis. 14.ed. Washington: AOAC, 1984.

BOLTON, P.R.; HEATON, K.W.; BURROUGHS, L.F. The role of dietary fiber in satiety, glucose, and insulin: studies with fruit and fruit juice. Journal of Clinical Nutrition, v.34, p.211-217, 1981.

CARSON, K.J.; COLLINS, J.L.; PENFIELD, M.P. Unrefined, dried apple pomace as a potential food ingredient. Journal of Food Science, v.59, p.1213-1215, 1994.

CHEN, H.; RUBENTHALER, G.L.; LEUNG, H.K.; BARANOWSKI, J.D. Chemical, physical, and baking properties of apple fiber compared with wheat and oat bran. Cereal Chemistry, v.65, p.244-247, 1988a.

CHEN, H.; RUBENTHALER, G.L.; SCHANUS, E.G. Effect of apple fiber and cellulose on the physical properties of wheat flour. Journal of Food Science, v.53, p.304-305, 1988b.

CUMMINGS, J.H. Nutritional implications of dietary fiber. American Journal of Clinical Nutrition, v.31, p.s21-s29, 1978.

DAVIES, J.R.; BROWN J.C.; LIVESEY, G. Energy values and energy balance in rats fed on suplements of guar gum or cellulose. British Journal of Nutrition, v.65, p.415-433, 1991.

HANG, Y.D. Production of fuels and chemicals from apple pomace. Food Technology, v.3, p.115-117, 1987.

HERNANDEZ, T.; HERNANDEZ, A.; MARTINEZ, C. Concepto, propiedades y metodos de analisis. Alimentaria, v.4, p.19-30, 1995.

KROTKIEWSKI, M. Effect of guar-gum on body weight, hunger ratings and metabolism in obese subjects. British Journal of Nutrition, v.52, p.97-105, 1984.

MIKKELSEN, O.; MAKDANI, D.D.; COTTON, R.H.; TITCOMB, S.T.; COLMEY, J.C.; GATTY, R. Effects of a high fiber bread diet on weightloss in college-age males. American Journal of Clinical Nutrition, v.32, p.1703-1709, 1979.

NAMIUCHI, N.N.; WOSIACKI, G.; ROCHA, M.A. Avaliação dos sucos de cinco variedades de maçãs nacionais. Características sensoriais dos sucos das variedades Fuji, Gala, Golden delicious, Granny Smith e Starkrimson. Revista Científica e Cultural da Universidade Federal do Mato Grosso do Sul, v.7, p.15-22, 1992.

OLIVEIRA, S.P.; REYES, F.G.R.; SGARBIERI, V.C.; AREAS, M.A; RAMALHO, A.C. Nutritional attributes of a sweet corn fibrous residue. Journal of Agricultural and Food Chemistry, v.39, p.740-743, 1991. 
PROSKY, L.; ASP, N-G; SCHWEIZER, T.F.; DeVRIES, J.W.; FURDA, I. Determination of insoluble, soluble and total dietary fiber in foods and food products: inter-laboratory study. Journal of the Association of Official Analytical Chemistrs, v.71, p.1017-1023, 1988.

PROTZEK, E.C.; FREITAS, R.J.S.; WASCZYNSKJ, N. Aproveitamento do bagaço de maçã na elaboração de biscoitos ricos em fibra alimentar. Boletim do Centro de Pesquisa e Processamento de Alimentos, v.16, p.263-275, 1998.

RAUPP, D.S.; SGARBIERI, V.C. Efeitos de frações fibrosas extraídas de feijão (Phaseolus vulgaris, L.) na utilização de macro e micronutrientes da dieta pelo rato. Ciência e Tecnologia de Alimentos, v.16, p.100-107, 1996.

RAUPP, D.S.; SGARBIERI, V.C. Efeito da fibra solúvel de alta viscosidade na ingestão de alimentos, na excreção fecal e no peso corpóreo, em ratos. Brazilian Archives of Biology and Technology, v.40, p.863-874, 1997.

RAUPP, D.S.; MOREIRA, S.S.; BANZATTO, D.A.; WOSIACKI, G.; SGARBIERI, V.C. Farinha de mandioca lite: industrialização potencial a partir de polpa de mandioca residual produzida como descarte pela polvilheira. In: CONGRESSO BRASILEIRO DE CIÊNCIA E TECNOLOGIA DE ALIMENTOS, 16., Rio de Janeiro, 1998. Anais. Rio de Janeiro: SBCTA, 1998. p.374.

RAUPP, D.S.; MOREIRA, S.S.; BANZATTO, D.A. Aproveitamento de resíduo resultante de indústrias alimentícias como fonte de nutrientes para humanos e animais: aproveitamento de resíduo resultante da industrialização da maçã. Ponta Grossa: Universidade Estadual, 1999. (Relatório de Projeto de Pesquisa Institucional)

REEVES, P.G.; NIELSEN, F.H.; FAHEY JUNIOR, G.C. AIN-93 Purified diets for laboratory rodents: final report of the American Institute of Nutrition Ad Hoc Writing Committee on the reformulation of the ain- $76^{A}$ rodent diet. The Journal of Nutrition, v.123, p.1939-1951, 1993.

RENARD, C.M.G.C.; THIBAULT, J.F. Composition and physicochemical properties of apple fibres from fresh fruits and industrial products. Lebensmittel Wissenschaft und Technologie, v.24, p.523-527, 1991.

ROEHRIG, K. The physiological effects of dietary fiber. Food Hydrocolloids, v.2, p.1-18, 1988.

SAURA CALIXTO, F. Fibra dietetica de manzana: Hacia nuevos tipos de fibras de alta calidad. Alimentaria, v.5, p.57-61, 1993.
SCHNEEMAN, B.O. Soluble vs insoluble fiber: different physiological responses. Food Technology, v.2, p.81-82, 1987.

SCHWEIZER, T.F.; EDWARDS, C.A. Dietary fibre: a component of food; nutritional function in health and disease. London: Springer-Verlag, 1992. p.3-20;103-136;249-332.

SEVA-PEREIRA, A.; MORAES, G.R.; OLIVEIRA, S.P.; REYES, F.G.R. Uso de biscoito rico em fibras no tratamento da constipação intestinal crônica. Revista Paulista de Medicina, v.109, p.265-268, 1991.

SMITH, A.N.; DRUMMOND, E.; EASTWOOD, M.A. The effects of coarse and fine Canadian Red Spring Wheat and French Soft Wheat bran on colonic motility in patients with diverticular disease. American Journal of Clinical Nutrition, v.34, p.2460-2463, 1981.

SOMOGYI, M. A new reagent for the determination of sugar. The Journal of Biological Chemistry, v.160, p.61-68, 1945.

TOMA, R.B.; CURTIS, D.J. Dietary fiber: effect on mineral bioavailability. Food Technology, v.2, p.111-116, 1986.

WALKER, A.R.P. Effect of high crude fiber intake on transit time and the absorption of nutrients in South African Negro schoolchildren, American Journal of Clinical Nutrition, v.28, p.1161-1169, 1975.

WALTER, R.H.; RAO, M.A.; SHERMAN, R.M.; COOLEY, H.J. Edible fibers from apple pomace. Journal of Food Science, v.50, p.747-749, 1985.

WANG, H.J.; THOMAS, R.L. Direct use of apple pomace in bakery products. Journal of Food Science, v.54, p.618-620, 1989.

WOSIACKI, G.; NAMIUCHI, N.N.; CERIBELLI, M.I.P.F.; SATAQUE, E.Y.; SICHIERI, V.L.F.S.; OLIVEIRA, T.C.R.M.; OLIVEIRA, C.E. Estabilidade do suco clarificado de maçã. Parte I- Processo de obtenção do suco de maçã. Brazilian Archives of Biology and Technology, v.32, p.775-786, 1989.

WOSIACKI, G.; CHIQUETTO, N.C.; KIRCHNER, C.L. Brazilian apple production. Fruit Processing, v.3, p.277-285, 1993.

WOSIACKI, G.; DEMIATE, I.M.; CHERUBIM, R.A. Brazilian apple juice industry. Fruit Processing, v.5, p.130-133, 1995.

WOSIACKI, G.; CHERUBIM, R.A.; HILGEMBERG, C. Characterization of apple juice of some new varieties growing in Brazil. Fruit Processing, v.8, p.306-310, 1996.

$\overline{\text { Recebido em } 16} .11 .99$ 